\title{
Programming Integrated Surgical Operations and Preventive Maintenance Activities
}

\author{
Michel Gourgand ${ }^{1}$, Janvier Pensi ${ }^{1,2}$, and Alain Tanguy ${ }^{1}$ \\ ${ }^{1}$ LIMOS CNRS UMR 6158-Université Blaise Pascal-Campus des Cézeaux, \\ 63173 Aubière Cedex, France \\ \{michel.gourgand, janvier.pensi, alain.tanguy\} @isima.fr \\ ${ }^{2}$ Faculty of Industrial Engineering, Douala University, Cameroon
}

\begin{abstract}
The operating theatre (OT) represents a significant component of the technical means centre. This facility is the largest cost and revenue centre. To be efficient, it needs an optimal operational pro- gramme, which takes into account maintenance activi- ties and not only surgical operations. To build such a programme, various methods have been used: mixed integer programming (MIP), three classic heuristics for Bin-Packing and a coupling of the first alterna- tive with a stochastic descent (SD). Then we compare the obtained results from generated data.
\end{abstract}

Keywords: Surgical unit, operating planning, MIP, heuristics.

\section{$1 \quad$ Introduction}

The renovation of the hospital centre: Laquintinie Hospital of Douala (LHD) in Cameroon has started for a few months with the aim to improve work conditions in the medical system environments. It was launched by the Cameroonian public authorities. Hospital team manager wants to reduce cost and to maximize the level of patient satisfaction. The OT was firstly chosen, to be optimized. Researchers having carried out works on the OT are unanimous: it is an important care provider, which generates large incomes and it is among the most important sources of expenditure. It is consuming between $10 \%$ and $15 \%$ of the hospital budget [1]. Hospital is generally organized in centres of responsibilities [2]. Each one is divided into functional units. Hospital is thus a complex structure, where various functions are provided by a multifield corporation. Managing the OT is hard due to the conflicting priorities and the preferences of its stakeholders [3], but also due to highly stochastic OT activities, such as the breakdown of equipment in the operating rooms. The actual realization of the planning and scheduling cannot be perfectly predicted. To improve management of the OT, this randomness has, to be taken into account. The problems the OT are known for the greatest part, and researchers proposed tools of decision-making aid, based on modelling, simulation and optimization. In this paper, we propose a tool to help OT managers in LHD, to improve the realization of the directing programme of surgical operations (DPSO), which takes into account surgical operations but also preventive maintenance activities (MA).

B. Grabot et al. (Eds.): APMS 2014, Part II, IFIP AICT 439, pp. 699-706, 2014.

(C) IFIP International Federation for Information Processing 2014 
The paper is organized as follow: in the next section, we review the related literature. The third section describes the problem to be handled. In the fourth section, we describe our model. The fifth section presents the generated data and the compared results, finally, we conclude and discuss extensions.

\section{State of the Art}

The aim of this literature research is to guide the OT managers in programming (planning and scheduling) this facility, by taking into account the impact of equipment breakdowns, on functioning and the benefice of the equipment maintenance. In the 60 last years, researchers focused on the OT management. Regarding planning and scheduling within the OT, [4] present a substantive state of the art survey. What the planning concerns, its resolution is quite not easy considering the number of constraints, namely: the intervention duration, the emergencies, the material availability, etc. In this section, we focus on proposed models and methods to solve problems having stochastic aspects. In the related literature, we met the following approaches: mixed integer programming (MIP), heuristics, simulation and Markov theory.

MIP method has been applied in various OT studies, with the aim to optimize the management of this facility. Most of the studies are concerned with the optimization of costs. For instance, minimization of costs of overused or underused operating rooms [5], or with the maximization of the daily benefit [6]. Authors focus on the minimization of the allocated time slots of surgeons [7]. To build a stable and flexible operational programme, others [8] applied MIP method so as to maximize the use of operating rooms.

Heuristics are more and more used. To minimize the costs of the intervention hours, [9] designed a genetic algorithm coupled with a procedure implementing a taboo search. In [10], they used heuristics after they identified the problem as a two level Flow-Shop, they minimized the overtime costs in the recovery rooms.

The simulation approach has been used to investigate particular cases, taking advantage of its main asset, i.e., the ability to model specific systems in details. In [11], a Monte Carlo simulation was coupled with a heuristics to maximize the room occupation rate and to minimize the overtime. Authors proposed a planning method to solve emergency problem [12], they combined a Monte Carlo simulation with MIP, to minimize overtime costs and the operation day costs.

Markov chains have been applied to health care management in various studies too. In [13], they proposed an analytical approach to evaluate the risks in the OT, to determine the number of operations which must be planned, in order to limit over- time.

Concerning the equipment maintenance, industrial managers are unanimous: the zero risk does not exit unfortunately, because human and material failures ever occur.

We focus works that proposed methods for solving maintenance activity problem and its impact to production activities. Several approaches have been developed to address MA problems. In [14], they used mathematical models solved with LINGO and MATLAB, to optimize integrated planning, taking into account 
preventive MA in production. In industrial systems, production programme could not be designed with- out regarding MA. This reality must be regarded too in health care systems. We have not found any papers solving together OT optimization and MA. In the present work, we proposed a model that combines the flows of planned surgical operations ( $\mathrm{SO})$ and preventive MA.

\section{Problem to Be Handled}

The research originates from collaboration with a Cameroonian hospital in the Douala area, from which we will receive real data that can be used to evaluate the reliability of our approach and illustrate its application. This hospital is composed of three surgical units: a gynaecologist-obstetrics unit, an emergencies unit and an OT, this OT is the object of our study. In this hospital, the OT is open five days a week (except for emergencies), for 8 hours a day. It can happen that the duration of an operation overflows closing time. The OT comprises six operating rooms (OR). Four rooms are dedicated to visceral surgery and urology, orthopaedic surgery, orthopaedic surgery and neurosurgery, and endoscopic surgery; the two other rooms are reserved for emergency cases. They are open 24 hours a day. But in this present work, we consider that all those as versatile rooms (able to accommodate any type of surgery). Each OR has his team consists of: 2 nurses, 1 instrumentalist, 1 anesthetist nurse, 1 team leader, 1 health care assistant, 1 anaesthetist doctor who supervises two OR. If the OR is not in service, the team is going to serve in other units. According to the operation, the team is supplemented by a surgeon and his assistant. The number of operations per OR and day does not exceed 4 (except for emergencies). After one operation, the staff has 30 minutes rest. The average time of decontamination is 25 minutes. To realize a daily scheduling in the OR, some criteria are applied: medical or clinical ones and availability of equipment.

\section{Approach}

In order to construct the DPSO model, some hypotheses are made: the open strategic planning and scheduling are used, DPSO is focusing only on the OR, the operation day is communicated to each patient, there is the possibility to postpone one planned operation, no criterion is considered to appointing patients, the equipment in the OR is only taken into account, emergencies are not regarded, the operation duration is estimated; health care units, induction and recovery rooms are well sized. We propose a tool to help OT managers in assessing the impact of equipment breakdown by planning DPSO. The aim is to help them, by guiding their decisions at an operational tactical level. We have to assign of MA and SO, it is a similar problem to that of Bin-Packing. It is a NP-hard problem. This problem is solved by three heuristics, a MIP model and the first heuristic variant coupled with a stochastic descent. We established first the algorithms First Fit (FT), Next Fit (NF) and Best Fit (BF). An MA can be planned at the opening time, either just before closing or in midday. So the day of 
medical use of a room can be cut in one or two periods. The approach is described as follows:

- Insert the MA on the weekly schedule: indicate the day, the room, the starting hour and the end hour of every MA

- For every day

- Operating day planning: indicate the day, the room and the period 1 or 2;

- Daily sequencing of the SO and the appointment of the surgeons: indicate the room and the time slot, postpone the SO in conflict the next day.

- Publishing of the weekly schedule and the untreated SO

The heuristics principle ones is to affect MA and SO respecting various constraints. We applied followings heuristic variants:

- Heuristics 1 (H1) affects SO in arrival order, according to the ascending order of the compatible rooms, days and period duration, without exceeding the maximum period use (First Fit Decreasing).

- Heuristics 2 (H2) proceeds initially by sorting SO in decreasing order of duration, then to their assignment (Next Fit Decreasing).

- Heuristics 3 (H3) proceeds by sorting SO in decreasing order of duration. Every day and before assignment, rooms are maintained sorted in ascending order of the remaining period duration. It assigns an SO to a compatible room period having the smallest availability duration (Best Fit).

\subsection{Mathematical Formalization}

Only one mathematical model is presented, because various mathematical formalizations of the Bin-Packing problem, in one dimension are similar.

Data, parameter and variable used in the model for the main problem are

NR number of operating rooms

$N D \quad$ number of days

NSO number of surgical operations

$N M A \quad$ number of maintenance activities

$D C_{c} \quad$ duration of $\mathrm{SO} c$ (including decontamination)

$D M_{m} \quad$ duration of MA $m$ (including cleaning-up time)

$Q \quad$ duration of the room opening $(8 \mathrm{~h})$

$c \quad$ index of surgical operations

$r \quad$ index of operating rooms

$d \quad$ index of working days of the horizon

$m \quad$ index of maintenance activities

$Z_{m r d} \quad 1$ if MA $m$ is allocated the $d$ day in room $r$; otherwise $=0$

$X_{c r d} \quad 1$ if SO $c$ is allocated the $d$ day in room $r$; otherwise $=0$ 


\section{$\underline{\text { Constraints }}$}

Respect of the room opening duration

$$
\left(\sum_{c=1}^{N S O} D C_{c} * X_{c r d}+\sum_{m=1}^{N M A} D M_{m} * Z_{m r d}\right) \leq Q ; r=1 \ldots N R, d=1 \ldots N D
$$

$\mathrm{SO} c$ is allocated in only one room and in only one day

$$
\sum_{r=1}^{N R} \sum_{d=1}^{N D} X_{c r d} \leq 1 ; c=1 \ldots N S O
$$

$\underline{\text { Parameter verifier }}$

MA $m$ is allocated in only one room and in only one day

$$
\sum_{r=1}^{N R} \sum_{d}^{N D} Z_{m r d} ; m=1 \ldots N M A
$$

$\underline{\text { Integrity constraints }}$

$$
X_{c r d}, Z_{m r d} \in\{0,1\} ; c=1 \ldots N S O, r=1 \ldots N R, d=1 \ldots N D, m=1 \ldots N M A
$$

Multi-objectives function

We have first to minimize the number of not allocated SO (NNASO), then the number of used room-day couples $(N U R D C)$ and finally, the minimum activity time $(M A T)$

$$
N N A S O=N S O-\sum_{c=1}^{N S O} \sum_{r=1}^{N R} \sum_{d=1}^{N D} X_{c r d}
$$

A Boolean function

$$
\begin{gathered}
Y_{r d}=\left(\sum_{c=1}^{N S O} X_{c r d}>0\right) \in\{0,1\}, r=1 \ldots N R, d=1 \ldots N D \\
N U R D C=\sum_{r=1}^{N R} \sum_{d=1}^{N D} Y_{r d} \\
M A T=\min _{r, d}\left(\sum_{c=1}^{N S O} X_{c r d} * D C_{c}+\left(1-Y_{r d}\right) * 10\right)
\end{gathered}
$$

The following multi-criterion objective results from the three ones $(M C O)$

$$
M C O=(N N A S O * 100+N U R D C) * 10+M A T
$$

\section{$5 \quad$ Results}

\subsection{Generated Data}

We illustrate the obtained results from generated data. It is composed of 15 preventive MA and 80 SO. Table 1 indicates following information: for the MA (number, type, start hour, end hour, room, day and the worker). Table 2 contains the data of SO: number, type and duration $(\mathrm{Du})$. The decontamination time is 25 minutes. All durations are given in minutes. 
Table 1. The MA data

\begin{tabular}{|c|c|c|c|c|c|c|}
\hline MAnum & MAtype & Sh & Eh & Room & Day & Worker \\
\hline 1 & 2 & 0 & 45 & 1 & 1 & $\mathrm{X} 1$ \\
\hline 2 & 1 & 180 & 240 & 2 & 1 & $\mathrm{X} 2$ \\
\hline 3 & 4 & 450 & 480 & 3 & 1 & $\mathrm{X} 3$ \\
\hline 4 & 5 & 0 & 60 & 2 & 2 & $\mathrm{X} 4$ \\
\hline 5 & 7 & 240 & 300 & 4 & 2 & $\mathrm{X} 1$ \\
\hline 6 & 3 & 0 & 30 & 3 & 2 & $\mathrm{X} 2$ \\
\hline 7 & 6 & 90 & 140 & 4 & 3 & $\mathrm{X} 3$ \\
\hline 8 & 6 & 90 & 140 & 1 & 3 & $\mathrm{X} 1$ \\
\hline 9 & 3 & 0 & 30 & 3 & 3 & $\mathrm{X} 4$ \\
\hline 10 & 2 & 429 & 474 & 1 & 4 & $\mathrm{X} 3$ \\
\hline 11 & 1 & 0 & 60 & 2 & 4 & $\mathrm{X} 2$ \\
\hline 12 & 7 & 420 & 480 & 4 & 4 & $\mathrm{X} 1$ \\
\hline 13 & 5 & 120 & 180 & 2 & 5 & $\mathrm{X} 4$ \\
\hline 14 & 5 & 0 & 60 & 3 & 5 & $\mathrm{Y} 1$ \\
\hline 15 & 4 & 300 & 350 & 4 & 5 & $\mathrm{Y} 2$ \\
\hline
\end{tabular}

\subsection{Mixed Integer Programming}

Firstly, we generalized the mathematical model so as to deal with room-dayperiods. Then we used two tools [15] (Gl and Gu) and [16] (Lp). Gl (Glpsol) and Gu (Gusek) used different solving options. Table 3 presents results with 60 seconds limited resolution duration. It indicates the number of allocated SO (Naso) and the room utilization duration (Rud). The first objective is studied using a MIP model solved with [15] and [16]. The multi-criterion objective is studied using a classic stochastic descent meta-heuristic coupled with a heuristic variant.

Table 2. The SO data

\begin{tabular}{|c|c|c|c|c|c|c|c|c|c|c|c|}
\hline Sonum & Sotvpe & $\mathrm{Du}$ & Sonum & Sotvpe & $\mathrm{Du}$ & Sonum & Sotvpe & $\mathrm{Du}$ & Sonum & Sotvpe & $\mathrm{Du}$ \\
\hline 1 & 3 & 90 & 21 & 10 & 90 & 41 & 3 & 90 & 61 & 8 & 120 \\
\hline 2 & 1 & 120 & 22 & 8 & 120 & 42 & 5 & 150 & 62 & 5 & 150 \\
\hline 3 & 2 & 90 & 23 & 9 & 120 & 43 & 7 & 90 & 63 & 4 & 120 \\
\hline 4 & 4 & 120 & 24 & 5 & 150 & 44 & 4 & 120 & 64 & 9 & 120 \\
\hline 5 & 5 & 150 & 25 & 6 & 90 & 45 & 2 & 90 & 65 & 9 & 120 \\
\hline 6 & 10 & 90 & 26 & 2 & 90 & 46 & 1 & 120 & 66 & 1 & 120 \\
\hline 7 & 8 & 120 & 27 & 1 & 120 & 47 & 8 & 120 & 67 & 1 & 120 \\
\hline 8 & 6 & 90 & 28 & 4 & 120 & 48 & 9 & 120 & 68 & 2 & 90 \\
\hline 9 & 7 & 90 & 29 & 3 & 90 & 49 & 10 & 90 & 69 & 3 & 90 \\
\hline 10 & 9 & 120 & 30 & 7 & 90 & 50 & 6 & 90 & 70 & 10 & 90 \\
\hline 11 & 1 & 120 & 31 & 5 & 150 & 51 & 4 & 120 & 71 & 2 & 90 \\
\hline 12 & 2 & 90 & 32 & 1 & 90 & 52 & 1 & 120 & 72 & 5 & 150 \\
\hline 13 & 8 & 120 & 33 & 4 & 120 & 53 & 8 & 120 & 73 & 4 & 120 \\
\hline 14 & 9 & 120 & 34 & 2 & 90 & 54 & 3 & 90 & 74 & 8 & 120 \\
\hline 15 & 3 & 90 & 35 & 3 & 90 & 55 & 5 & 150 & 75 & 10 & 90 \\
\hline 16 & 5 & 150 & 36 & 6 & 90 & 56 & 6 & 90 & 76 & 3 & 90 \\
\hline 17 & 4 & 120 & 37 & 7 & 90 & 57 & 6 & 90 & 77 & 2 & 90 \\
\hline 18 & 10 & 90 & 38 & 10 & 90 & 58 & 8 & 120 & 78 & 6 & 90 \\
\hline 19 & 7 & 90 & 39 & 8 & 120 & 59 & 7 & 90 & 79 & 4 & 120 \\
\hline 20 & 6 & 90 & 40 & 9 & 120 & 60 & 7 & 90 & 80 & 5 & 150 \\
\hline
\end{tabular}


Table 3. MIP results

\begin{tabular}{|c|c|c|c|c|c|c|}
\hline Horizon & \multicolumn{2}{|c|}{ Gl } & \multicolumn{2}{c|}{ Gu } & \multicolumn{2}{c|}{ Lp } \\
\hline Day & Naso & Rud & Naso & Rud & Naso & Rud \\
\hline 1 & 21 & 2415 & 21 & 2505 & 21 & 2415 \\
\hline 2 & 38 & 4550 & 42 & 5010 & 38 & 4580 \\
\hline 3 & 57 & 7155 & 58 & 7390 & 55 & 7075 \\
\hline 4 & 71 & 9335 & 74 & 9890 & 72 & 9570 \\
\hline 5 & 80 & 10700 & 80 & 10700 & 80 & 10700 \\
\hline
\end{tabular}

\subsection{Heuristics Results}

Table 4 presents obtained results with the three heuristics. It gives Naso, the number of used room-days (Nurd) and the number of room day periods Nrdp).

Table 4. Heuristics results

\begin{tabular}{|c|c|c|c|c|c|c|c|c|c|c|}
\hline Horizon & \multicolumn{3}{|c|}{$\mathrm{H} 1$} & \multicolumn{3}{|c|}{$\mathrm{H} 2$} & \multicolumn{3}{|c|}{$\mathrm{H} 3$} & SD \\
\hline Day & Naso & Nurd & Nrdp & Naso & Nurd & Nrdp & Naso & Nurd & Nrdp & Naso \\
\hline 1 & 15 & 6 & 7 & 17 & 6 & 7 & 17 & 6 & 7 & 21 \\
\hline 2 & 32 & 12 & 14 & 35 & 12 & 14 & 34 & 12 & 14 & 40 \\
\hline 3 & 49 & 18 & 20 & 51 & 18 & 20 & 51 & 18 & 20 & 57 \\
\hline 4 & 70 & 24 & 26 & 72 & 24 & 27 & 68 & 24 & 26 & 75 \\
\hline 5 & 80 & 27 & 30 & 80 & 27 & 31 & 80 & 29 & 33 & 80 \\
\hline
\end{tabular}

$\mathrm{H} 1$ does not affect SO better than $\mathrm{H} 3$ and $\mathrm{H} 2$. $\mathrm{H} 2$ affects more and uses in less Nurd than H3. When all SO are allocated, horizon 5, H1 uses less Nurd and less Nrdp than the other heuristics. In MIP, the options used for Gu allocate more SO than Gl, Lp and the three heuristics. The meta-heuristic SD generates scheduling and optimizes the criteria. H1 evaluates them for the tested scheduling. SD gives the best results.

\section{Conclusion and Extensions}

We proposed a mathematical model for our problem and we implemented and applied exact methods and estimations on generated data. The obtained results are satisfying, especially the multi-criterion stochastic descent. Our tool of decision-making aid will be used in the hospital on real data. The prospects concern the surgical unit programming, the SO planning, the allocation of material resources and the management of the curative maintenance activities. 


\section{References}

1. Macario, A., Vitez, T.S., Dunn, B., Mcdonald, T.: Where Are the Costs in Perioperative Care?: Analysis of Hospital Costs and Charges for Inpatient Surgical Care. Anesthesiology 83, 1138-1144 (1995)

2. Combes, C.: Un environnement de modélisation pour les systèmes hospitaliers. Thèse de Doctorat, Université Blaise Pascal, France (1994)

3. Glauberman, S., Mintzberg, H.: Managing the care of health and the cure of disease. Part I: Differentiation. Health Care Management Review 26, 56-69 (2001)

4. Cardoen, B., Demeulemeester, E.: Operating room Planning and scheduling: A literature review. European Journal of Operational Research 201, 921-932 (2010)

5. Jebali, A., Hadj Alouane, A., Ladet, P.: Operating rooms scheduling. International Journal of Production Economics 99, 52-62 (2006)

6. Marcon, E., Kharraja, S., Simonnet, G.: The operating theatre scheduling:an approach centred on the follow-up of the risk of no realization of the planning. In: Proceeding of the Industrial Engineering and Production Management, Canada, pp. 18-21 (2001a)

7. Hammami, S., Ladet, P., Atidel, B.H.A., Ruiz, A.: Une programmation opératoire robuste. Logistiqueet Management 15, 95-111 (2007)

8. Agnetis, A., Coppi, A., Corsini, M., Dellino, M.C., Pranzo, M.: Longterm evaluation of operating theater planning policies. Operations Research for HealthCare 1, 95-104 (2012)

9. Fei, H., Duvivier, D., Meskens, N., Chu, C.: Ordonnancement journalier dans unbloc opératoire danslecadred'unestratégie openscheduling. In: Gestionet Ingénieriedes Systèmes Hospitaliers (GISEH 2006), Luxembourg (2006)

10. Iser, J.H., Denton, B.T., King, R.E.: Heuristics for balancing operating room and postanesthesia resources under uncertainty. In: Winter Simulation Conference, Miami, USA, pp. 1601-1608 (2008)

11. Hans, E., Wullink, G., Van Houdenhoven, M., Kazemier, G.: Robust surgery loading. European Journal of Operational Research 185, 1038-1050 (2008)

12. Lamiri, M., Xie, X., Dolgui, A., Grimaud, F.: Astochastic model for operating room planning with elective and emergency demand for surgery. European Journal of Operational Research 185, 1026-1037 (2008)

13. Tancrez, J.-S., Roland, B., Cordier, J.P., Riane, F.: Assessing the impact of stochasticity for operating theater sizing. Decision Support Systems 55, 616-628 (2013)

14. Fitouhi, M.C.: Optimisation de la planification intégrée de la maintenance préventive et de la production des systèmes multi-états. Thèse de Doctorat, Université Laval, Québec (2011)

15. Glpk (2014), http: / / www . gnu . org/software/glpk/

16. Lpsolve (2014), http: / / lpsolve. sourceforge.net/5.5/ 